\title{
Probing Local Structures and Disorder in Graphitic Carbon Nitrides
}

\author{
Diane M. Haiber ${ }^{1 *}$, Barnaby D.A. Levin ${ }^{1}$ and Peter A. Crozier ${ }^{1}$ \\ 1. School for Engineering of Matter, Transport \& Energy, Arizona State University, Tempe, AZ, USA \\ *dhaiber@asu.edu
}

Graphitic carbon nitrides $\left(\mathrm{g}-\mathrm{CN}_{\mathrm{x}} \mathrm{H}_{\mathrm{y}} \mathrm{s}\right)$ have emerged as promising candidates for driving photocatalytic $\mathrm{H}_{2}$ evolution under visible light, $\mathrm{CO}_{2}$ reduction, and other renewable energy applications owing to their native high surface area, bandgap in the visible regime, and inexpensive synthesis routes [1]. Calcination of various N-rich precursors yields layered compounds with interplanar spacings of $\sim 3.2 \AA$. Although commonly referred to as " $\mathrm{g}-\mathrm{C}_{3} \mathrm{~N}_{4}$ ", the average structure of $\mathrm{g}-\mathrm{CN}_{\mathrm{x}} \mathrm{H}_{\mathrm{y}} \mathrm{s}$ are consistent with polymeric melon $\left(\mathrm{C}_{2} \mathrm{~N}_{3} \mathrm{H}\right)$ whose in-plane structure is composed of amine $\left(\mathrm{N}-\mathrm{H}_{\mathrm{x}}\right)$ bridged heptazine $\left(\mathrm{C}_{6} \mathrm{~N}_{7}\right)$ building blocks (Figure 1a) [2]. However, structural differences between different $\mathrm{g}-\mathrm{CN}_{\mathrm{x}} \mathrm{H}_{\mathrm{y}} \mathrm{s}$ are not well understood as X-ray diffraction (XRD) and related measurements are limited to the bulk. High spatial resolution techniques, such as transmission electron microscopy (TEM), may be leveraged to probe the in-plane structure/disorder in these materials.

Aberration-corrected TEM at $300-\mathrm{kV}$ under low fluence rate conditions $\left(<70 \mathrm{e}^{-} / \AA^{2} / \mathrm{s}\right)$, enabled by the use of a direct electron detector (K2-IS, Gatan), was applied to three $\mathrm{g}-\mathrm{CN}_{\mathrm{x}} \mathrm{H}_{\mathrm{y}} \mathrm{s}$ demonstrating different degrees of structural condensation. The extent of long-range order in each sample was ranked based on the relative Bragg peak broadening observed in XRD patterns. Nicanite (a commercially produced g$\left.\mathrm{CN}_{\mathrm{x}} \mathrm{H}_{\mathrm{y}}\right)$ exhibits the most long-range order compared to the two urea-derived samples $\left(\mathrm{U}_{240}-\mathrm{gCN}\right.$ and $\left.\mathrm{U}_{30}-\mathrm{gCN}\right)$. $\mathrm{U}_{240^{-}} \mathrm{gCN}$ and $\mathrm{U}_{30}-\mathrm{gCN}$ were produced by calcining urea at $550^{\circ} \mathrm{C}$ for 240 and 30 minutes, respectively, yielding varied degrees of condensation. Large field of view $(\sim 70 \mathrm{~nm})$ images of each sample were then obtained by imaging regions wherein the extended layers were perpendicular to the incident electron beam. Figure $1 \mathrm{~b}$ shows a small, $10-\mathrm{nm}$ region from $\mathrm{U}_{240}-\mathrm{gCN}$ as an example. Fourier transforms (FTs) generated from these images exhibit exceptional spatial resolution which is evidenced by the multiple, well-resolved $(h k 0)$ reflections present out to $(1 \AA)^{-1}$ in Nicanite. Figure 1 c compares the radially-averaged FT from $\mathrm{U}_{240}-\mathrm{gCN}$ to its XRD pattern. When the radially-averaged FTs are compared to the corresponding XRD data, a similar trend in Bragg peak broadening is observed which suggests that the low fluence rates used for TEM imaging have avoided significant structural degradation.

To visualize the local heptazine-heptazine nearest neighbor (HHNN) environment, each large field of view TEM image was broken up into a grid of 6.5-nm boxes. A 2D autocorrelation was computed for each sub-region, each of which represent the local pseudo Patterson function (PPF) at a particular location within the imaged area. Figure 2a displays the mean PPF for $\mathrm{U}_{240}$-gCN. Positive-to-negative intensity oscillations represent the HHNN shells, which extend at least up to the $4^{\text {th }}$ shell in Nicanite. $\mathrm{U}_{240}$-gCN demonstrates strong structural correlations out to the $3^{\text {rd }}$ shell while $\mathrm{U}_{30}$-gCN exhibits structural ordering only out to the $1^{\text {st }}$ shell. Figure $2 \mathrm{~b}$ compares three local PPFs from $\mathrm{U}_{240}-\mathrm{gCN}$ wherein increased structural ordering was observed. In general, local PPFs extracted from these more ordered regions of the urea-derived $\mathrm{g}-\mathrm{CN}_{\mathrm{x}} \mathrm{H}_{\mathrm{y}} \mathrm{s}$ exhibit increased azimuthal orientational variations of the structural units which can be seen in Figure 2b. Collectively, these in-plane fluctuations manifest as circular symmetry in the mean PPFs. On the other hand, each local PPF from Nicanite resembles the mean PPF meaning that across the field of view, the in-plane structure remains relatively invariant. Virtual dark field images generated from the TEM image of Nicanite also reveals in-plane domains 
extending over 10's of nanometers. Overall, this analysis suggests that the average heptazine chain length in $\mathrm{g}-\mathrm{CN}_{\mathrm{x}} \mathrm{H}_{\mathrm{y}} \mathrm{s}$ can vary considerably. Shorter heptazine chains (approximately 2-4 units or 1-2 $\mathrm{nm}$ ) occur in the urea-derived compounds whereas much longer chains (10's of nanometers) are stabilized in Nicanite. Increased hydrogen content, leading to a greater fraction of chain-terminating $\mathrm{N}-\mathrm{H}_{2}$ groups most likely results in the shortened chains and allows for structural disorder to progress in the way observed here.

References:

[1] T.S. Miller et al., Phys. Chem. Chem. Phys 19 (2017), p. 15613.

[2] B.V. Lotsch et al., Chem. -A Eur. J. 13 (2007), p. 4969.

[3] We gratefully acknowledge the support from the DOE (DE-SC0004954), Gatan Inc. for the use of the K2-IS detector, ASU's John M. Cowley Center for High Resolution Electron Microscopy and ASU's Eyring Materials Center.
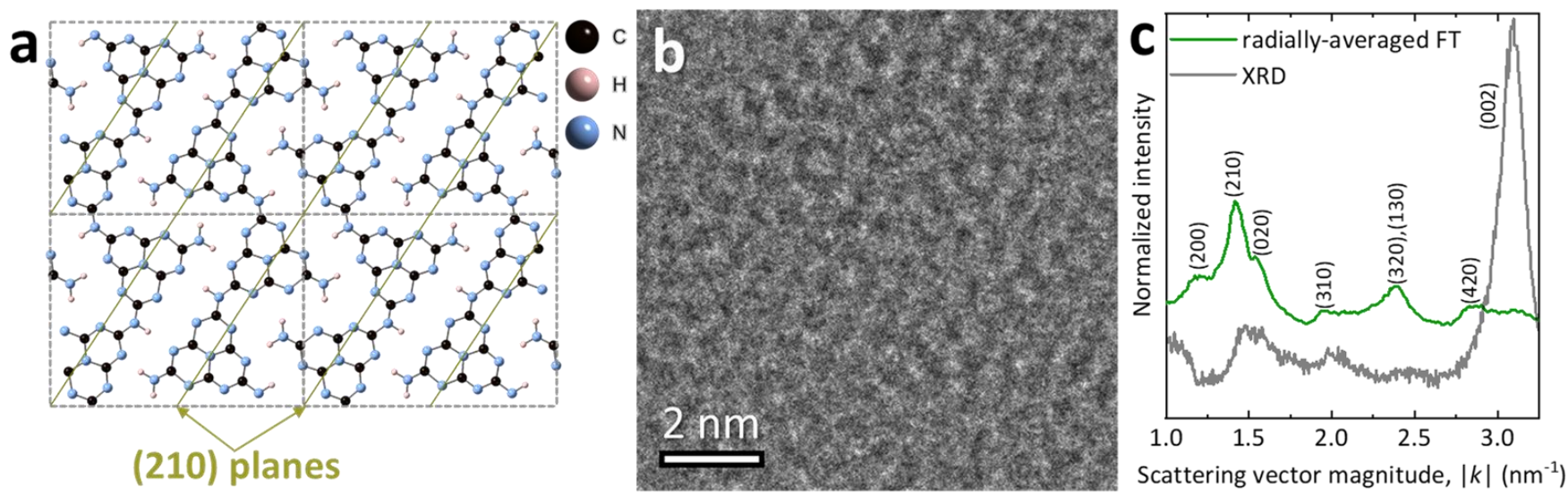

Figure 1. (a) Single-layer structure model for polymeric melon $\left(\mathrm{C}_{2} \mathrm{~N}_{3} \mathrm{H}\right)$ showing the (210) planes. (b) Low fluence-rate $\mathrm{HR}-\mathrm{TEM}$ image from $\mathrm{U}_{240} \mathrm{gCN}$, cropped from the larger field of view image. (c) Radially-average FT (generated from the entire field of view) compared to the XRD pattern for $\mathrm{U}_{240^{-}}$ $\mathrm{gCN}$.
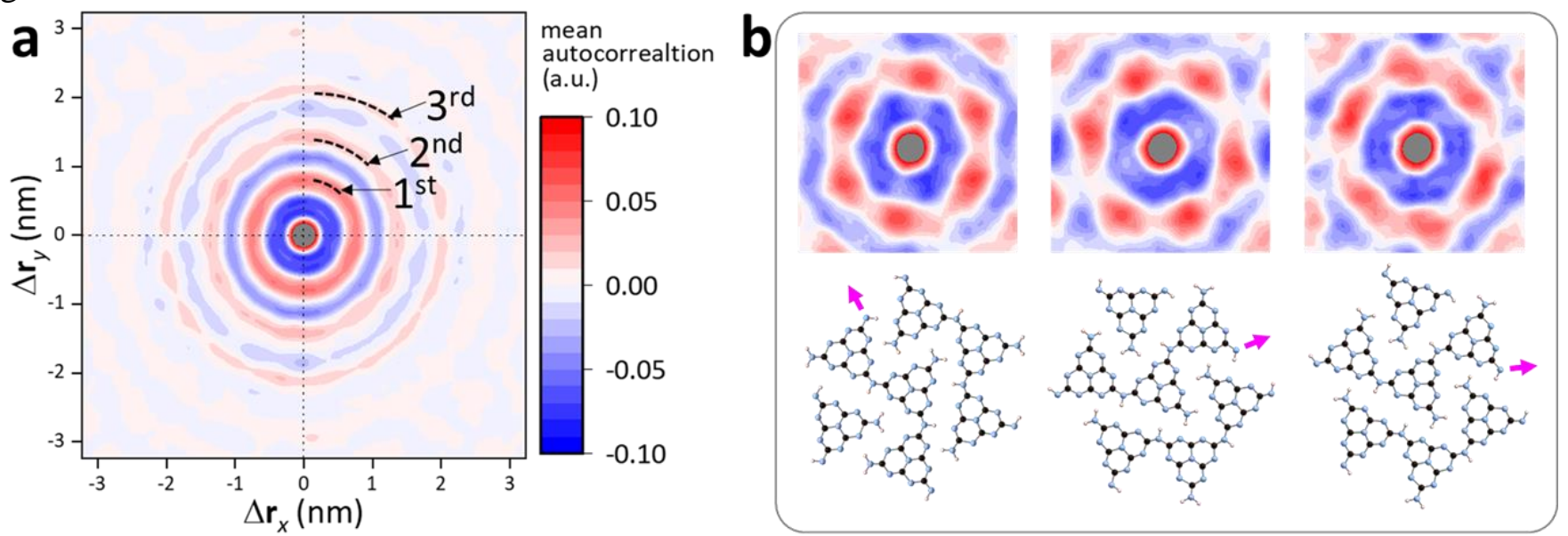

Figure 2. (a) Mean pseudo Patterson function (PPF) for $\mathrm{U}_{240} \mathrm{gCN}$; the $1^{\text {st }}-4^{\text {th }}$ heptazine-heptazine nearest neighbor (HHNN) shells are labelled over the mean PPF. (b) Examples of three local PPFs from $\mathrm{U}_{240}-\mathrm{gCN}$ exhibiting local structural ordering. Below each local PPF is a structure model containing the first HHNN shell depicting the azimuthal orientation in that region. The pink arrows are a guide to the eye to more clearly show the azimuthal orientation variation. 